\title{
Survey on Spectrum monitoring for OFDM based Cognitive Radio Networks
}

\author{
Julia Merin Lal ${ }^{1}$ Shiras S. N ${ }^{2}$ \\ ${ }^{I}$ Department of Electronics and Communication MBCET Trivandrum, India
}

\begin{abstract}
Cognitive radio is a novel approach for improving the utilization of the radio electromagnetic spectrum. When a frequency band has primary and secondary users, the cognitive radios of the secondary users must monitor the band and be ready to cease their transmissions if a primary user's radio begins to transmit. Conventional spectrum sensing requires the secondary radios to refrain from communicating while they check for the emergence of primary signals. This paper gives an overview of different spectrum monitoring approaches for OFDM based cognitive radio network.
\end{abstract}

Keywords: Cognitive radio, dynamic spectrum access, OFDM, spectrum sensing/monitoring

\section{Introduction}

The radio spectrum is becoming increasingly congested everyday with the increasing number of wireless devices. Also wide ranges of spectrum is rarely used where as other bands are heavily used. The unoccupied portions of the licensed spectrum can only be used by licensed users. CR system uses its gained experience to plan future actions and adapt to improve the overall communication quality and meet user's needs. Thus a CR can be defined as an intelligent wireless system that is aware of its surrounding environment through sensing and measurements. CR autonomously exploit locally unused spectrum to improve spectrum utilization. CR's ability to sense and be aware of its operational environment, and dynamically adjust its radio operating parameters accordingly can be achieved by making the physical layer (PHY) highly flexible and adaptable. one of the most widely used technologies in current wireless communications systems is a multicarrier transmission known as orthogonal frequency division multiplexing (OFDM).OFDM can overcome many problems that arise with high bit rate communications, the biggest of which is time dispersion. The symbol stream that bears data is split into several lower rate streams and these streams are transmitted on different carriers. This splitting increases the symbol duration by the number of orthogonally overlapping carriers (subcarriers), multipath echoes affect only a small portion of the neighboring symbols.

In overlay network model SUs opportunistically use the spectrum when PUs are idle. Both primary and secondary users cannot operate simultaneously. Here prior to communication secondary users must sense the spectrum and detect whether it is available or not. When the PU is idle the Su is allowed to use the spectrum but it has to monitor the shared band to vacate the band as soon as primary user reappearance is detected. The SU must be capable of determining very weak signal from primary user. In this process, the CR system may spend a long time which is known as sensing interval. During the sensing interval the secondary transmitters are silent while the frequency band is sensed. During the detection time the CR users does not utilize the spectrum and so it is known as Quiet periods(QPs)[4].In the IEEE 802.22 system a quiet period consists of a series of consecutive spectrum sensing intervals using energy detection and these are to determine whether the signal level is higher than a predefined value. If it is higher than the predefined value then it indicates a non-zero probability of primary user transmission. Followed by the energy detection there is a feature detection to distinguish whether the source of energy is primary user or noise [5],[6].This process is repeated periodically to monitor the spectrum. If a PU is detected then the SU vacates the spectrum for a finite period of time and selects another valid spectrum band in spectrum pool for communication. Here, SU stops communicating periodically to detect the reappearance of PU. There are mainly to effects to be considered in this case. If the underlying communication technique is sensitive to synchronization errors then the SU receiver may lose its synchronization to the SU transmitter and this reduces the overall degradation in the secondary network performance during quiet periods [7].Another effect is the degradation of Quality of service for real time applications like Voice over IP (VoIP) which is due to reduction of throughput of the secondary network during sensing intervals to zero [8]. If the duration of the sensing intervals is too large then the impact becomes more severe as the average throughput of the secondary network becomes very low. Whereas, if this duration is too small, then the spectrum sensing provides no information about the frequency band of interest between consecutive sensing intervals and therefore the interference to the primary users is increased. There have been efforts which attempts to minimize the time duration for spectrum monitoring by jointly optimizing the sensing time with the detection threshold[9].To protect the PU while the sensing time is minimized we consider the PU throughput statistics. In conventional systems ,before the SU communication traditional spectrum sensing is 
applied once and unless the monitoring algorithm indicates the presence of PU in the band spectrum sensing is not repeated. When the monitoring correctly determines that there is no primary signal in the band the time that would have been spent performing spectrum sensing can be used to deliver packets in the secondary network. Thus the spectrum efficiency of the secondary network is improved. When the spectrum monitoring detects a primary signal in the band during a time period in which spectrum sensing would not have been scheduled, the disruption to the primary user can be terminated more quickly. Thus the impact of secondary communications on the primary user is reduced. Thus the SU receiver must follow two consecutive phases, which are sensing phase and monitoring phase. Among both the phases sensing phase is applied over a predefined period.

In another approach the spectrum is monitored by the CR receiver during reception and without any quiet periods [2]. Here for each received packet, compare the bit error count which is produced by a strong channel code like a Low Density Parity Check (LDPC) code to a threshold value. The monitoring algorithm indicates that the primary user is active when the number of detected errors is above certain value. By considering the hypothesis test for the receiver statistics when the primary signal is absent and the receiver statistics for the desired Secondary-to-Primary power Ratio (SPR) the threshold value is obtained. This technique is simple and adds no complexity to the system. But the problem is that the receiver statistics are subject to change by varying the system operating conditions. The parameters that can affect the receiver error count in real systems are RF impairments including Phase Noise (PN) and Carrier Frequency Offset (CFO), Sampling Frequency Offset (SFO), and NBI. The error count will depend on the presence of a primary signal and characteristics of those impairments. Based on the residual errors generated from estimating and compensating for different impairments, the receiver statistics may change from one receiver to the other. Due to the difficulty in characterizing the receiver statistics for all CR receivers, it is better to devise an algorithm that is robust to synchronization errors and channel effects. OFDM is a reliable and effective transmission method which is a multi-carrier modulation technique that is used in many wireless systems. OFDM is utilized as the physical layer modulation technique for many wireless systems like DVB-T/T2, LTE, IEEE $802.16 \mathrm{~d} / \mathrm{e}$, and IEEE $802.11 \mathrm{a} / \mathrm{g}[3]$. OFDM is preferred for cognitive networks. It has been already in use for the current cognitive standard IEEE 802.22.

In the traditional spectrum monitoring techniques, which rely on the periodic spectrum sensing during quiet periods, apply their processing over the received time domain samples to explore a specific feature to the primary user. Further, it is totally appropriate to remove the quiet periods during the monitoring phase to improve the network throughput. The signal construction for the secondary user can help the spectrum monitoring to happen without utilizing QPs. If the secondary user utilizes OFDM as the physical transmission technique, then a frequency domain based approach can be employed to monitor the spectrum during the CR reception only if the SU transmitter adds an additional feature to the ordinary OFDM signal. A spectrum monitoring technique, namely the energy ratio (ER) technique, that is suitable for OFDM-based cognitive radio as in [1] can be used. In this technique the transmitter helps this frequency domain based spectrum monitoring approach by introducing scheduled null-tones by which the spectrum can be monitored during CR reception. It is designed to detect the reappearance of primary user which also uses OFDM techniques. This technique implies fast response to PU appearance since it operates over the OFDM signal chain and hence, it need not wait for the decoded bits.

\section{OFDM-BASED CR}

OFDM can overcome many problems that arise with high bit rate communications, the biggest of which is time dispersion. The symbol stream that bears data is split into several lower rate streams and these streams are transmitted on different carriers. This splitting increases the symbol duration by the number of orthogonally overlapping carriers (subcarriers), multipath echoes affect only a small portion of the neighboring symbols. By extending the OFDM symbol with a cyclic prefix (CP),remaining inter-symbol interference (ISI) is removed. By this method, OFDM reduces the dispersion effect of multipath channels encountered with high data rates and reduces the need for complex equalizers. The advantages of OFDM include high spectral efficiency, robustness against narrowband interference (NBI), scalability, and easy implementation using fast Fourier transform (FFT).

In [3], it is assumed that a CR system operate as a secondary user in a licensed band. The CR system senses available or unused parts of the spectrum and exploit them. Aim is to achieve maximum throughput while keeping interference to primary/licensed users to a minimum. In the IEEE 802.22 standard-based CR system, the spectrum allocated for TV channels is reused. Here, the TV channels are the primary users and the standard-based systems are the secondary users. The block diagram of the CR-OFDM system is shown in Fig. 1. 


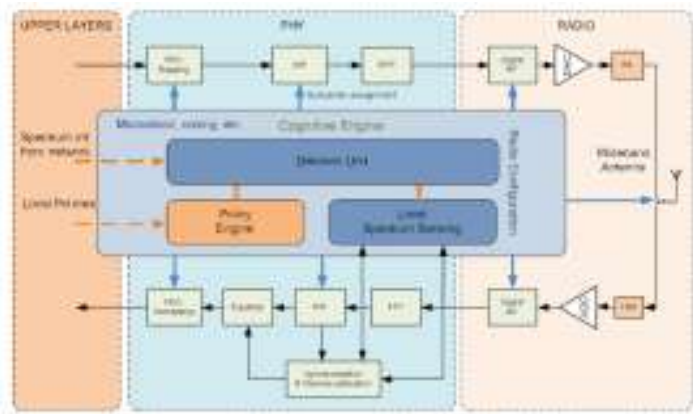

Fig. 1. OFDM-based CR system block diagram [3]

The cognitive engine makes intelligent decisions and configuring the radio and PHY parameters. Based on the information from policy engine as well as local and network spectrum sensing data, the transmission opportunities are identified by the decision unit. When the PHY layer is concerned, CR can communicate with various radio access technologies in the environment, or depending on the environmental characteristics it can improve the communication quality, by simply changing the configuration parameters of the OFDM system parameters) and the radio frequency (RF) interface. The coding type, coding rate, interleaver pattern, and other medium access control (MAC) and higher layer functionalities etc. should also be changed accordingly.

\section{SPECTRUM MONITORING APPROACHES}

In conventional systems, before the SU communication traditional spectrum sensing is applied once and unless the monitoring algorithm indicates the presence of PU in the band spectrum sensing is not repeated. When the monitoring correctly determines that there is no primary signal in the band the time that would have been spent performing spectrum sensing can be used to deliver packets in the secondary network. Thus the spectrum efficiency of the secondary network is improved. When the spectrum monitoring detects a primary signal in the band during a time period in which spectrum sensing would not have been scheduled, the disruption to the primary user can be terminated more quickly. Thus the impact of secondary communications on the primary user is reduced. Thus the SU receiver must follow two consecutive phases, which are sensing phase and monitoring phase. Among both the phases sensing phase is applied over a predefined period. The traditional spectrum monitoring techniques, which rely on the periodic spectrum sensing during quiet periods, apply their processing over the received time domain samples to explore a specific feature to the primary user. Further, it is totally appropriate to remove the quiet periods during the monitoring phase to improve the network throughput. The signal construction for the secondary user can help the spectrum monitoring to happen without utilizing QPs.

In receiver statistic approach the spectrum is monitored by the CR receiver during reception and without any quiet periods [2].Here for each received packet, compare the bit error count, which is produced by a strong channel code like a Low Density Parity Check (LDPC) code to a threshold value. The monitoring algorithm indicates that the primary user is active when the number of detected errors is above certain value. By considering the hypothesis test for the receiver statistics when the primary signal is absent and the receiver statistics for the desired Secondary-to-Primary power Ratio (SPR) the threshold value is obtained. This technique is simple and adds no complexity to the system. But the problem is that the receiver statistics are subject to change by varying the system operating conditions. The parameters that can affect the receiver error count in real systems are RF impairments including Phase Noise (PN) and Carrier Frequency Offset (CFO), Sampling Frequency Offset (SFO), and NBI. The error count will depend on the presence of a primary signal and characteristics of those impairments. Based on the residual errors generated from estimating and compensating for different impairments, the receiver statistics may change from one receiver to the other. Due to the difficulty in characterizing the receiver statistics for all CR receivers, it is better to devise an algorithm that is robust to synchronization errors and channel effects.

Another spectrum monitoring technique, namely the energy ratio (ER) technique, that is suitable for OFDM-based cognitive radio as in [1] can be used. In this technique the transmitter helps this frequency domain based spectrum monitoring approach by introducing scheduled null-tones by which the spectrum can be monitored during CR reception. It is designed to detect the reappearance of primary user which also uses OFDM techniques. This technique implies fast response to PU appearance since it operates over the OFDM signal chain and hence, it does not wait for the decoded bits.

\section{A. Receiver Statistics}

Receiver statistics is a physical-layer statistics that permit a cognitive radio to monitor the band in which it is receiving a packet. The statistics for each cognitive radio of the secondary network is derived in its 
demodulator and decoder. Here it is assumed that the secondary users are performing session based communications for which each session requires the delivery of several hundred to several thousand packets. And here, the secondary radios employ traditional spectrum sensing to identify an unused frequency band prior to initiating a session. But, they do not conduct traditional spectrum sensing during a session unless the receiver statistics suggest that a primary signal may be present in the band. The secondary radio that is sending the packets is named as the source and a secondary cognitive radio that is receiving the packets is named as a destination. Even if non-binary modulation is employed, binary codes are used for error control, to provide maximum flexibility. Transmitter and receiver models with receiver statistics are shown in fig 2 .

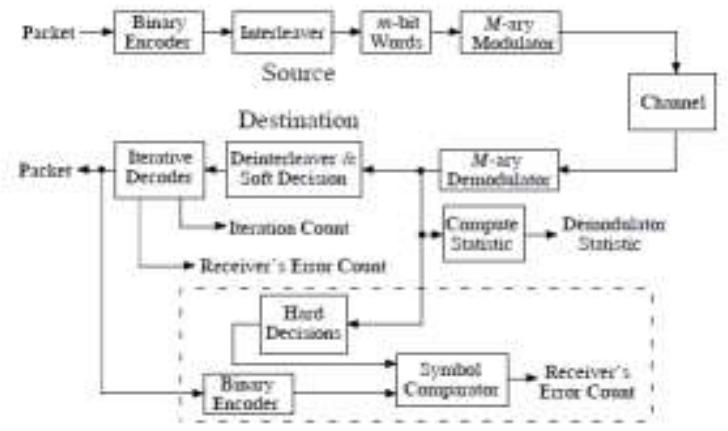

Fig 2. Transmitter and receiver models with receiver statistics[2].

The error-control codes in the system of Fig. 2 are binary convolution, turbo, and low-density parity check (LDPC) codes. Two data sequences are defined. The source's data sequence is formed from the binary symbols at the encoder output in the transmitter and the destination's data sequence is formed from the sequence of binary hard decisions at the output of the demodulator. The soft decision from the demodulator is used by the decoder whereas the hard decisions in the destination's data sequence are used for the receiver's error count. The sequence of information bits at the decoder output is identical to the information sequence that was sent by the source if the packet is decoded correctly, if the packet is decoded correctly. But even for a correctly decoded packet, the destination's data sequence may differ from the source's data sequence. The error count (EC) for the packet is the number of positions in which the two data sequences disagree. The receiver's error count (REC) is defined only for correctly decoded packets .Because when a packet does not decode correctly, then the receiver typically cannot determine the destination's data sequence, so it cannot determine the EC. The REC remains the same as the EC, for such packets. When a packet either fails to decode or decodes correctly but has a large value for the REC, then it is likely that the received signal was corrupted, perhaps by a transmission from a primary radio. For the destination, to determine the REC for a correctly decoded packet one way is to encode the information bits out of the decoder and compare the encoded sequence with the destination's data sequence. Some decoders, can provide the REC directly, in which case the encoder and comparator of Fig.2 are not needed. Examples are decoders such as sum-product algorithm (SPA) decoders for LDPC codes. The REC and the EC are identical for a correctly decoded packet. Whereas, only the EC is defined for a packet that is not decoded correctly.

The receiver statistic known as the iteration count (IC) can be employed to detect the emergence of a primary signal, when the secondary receivers employ iterative decoding. The total number of iterations required to decode all received words in the packet divided by the number of code words that were sent in the packet is the IC for a packet. When the IC is large for a packet, then it is likely that the received signal representing the packet was corrupted, and the corruption might be due to the presence of a primary signal in the frequency band. The conditional probability distribution of the REC given that there is no primary signal in the band is the quiescent distribution of the REC at a destination, and the conditional distribution given that there is a primary signal in the band is the active distribution, for each destination the shift in the distribution of the REC strongly indicate the presence of the primary signal. In EC-based monitoring, detection occurs when the emergence of the primary signal causes an increase in the value of the EC.As a result, the lowest probability of detection occurs for time and phase offsets that minimize the primary signal's interference effects on the destination receiver's binary symbol demodulation.

In monitoring based on the REC protocol employs receiver statistics that are obtained for each packet the source delivers to the destination. The destination must establish some characteristics of the receiver statistics that occurs in the channel's quiescent state, which is the state when there is no primary signal in the band. The characteristics could be learned during the demodulation and decoding of the first packet that is sent after it is determined by spectrum sensing that there is no primary signal in the band, for channels with only very small variations over the duration of a session, and then these characteristics can be used for the entire session. 
However for more dynamic channels, the characteristics should be obtained from each packet for which it is determined that there is no significant interference from other signals, and then these characteristics are used only for the next packet. Due to the uncertainties in the nature of the channel in practical applications, the latter approach is preferred because it gives more robust performance.

The destination must decide whether a primary signal is present, during the demodulation and decoding of each packet. Consider $H 0$ as the hypothesis that there is no primary signal in the band when the packet was received and $H 1$ be the hypothesis that there is a primary signal in the band. A receiver statistic from the most recent packet, is compared with a threshold determined by the destination is the decision statistic. The outcome of the comparison finds the decision that the destination makes: Decide $H 0$ or decide $H 1$. The conditional probability that the destination decides $H 1$ given that $H 1$ is true, is known as the detection probability $P d$ and the conditional probability that the destination decides $H 1$ given that $H 0$ is true is known as the false-alarm probability $P f$. The decision rule maximizes the detection probability subject to a constraint on the probability of false alarm, but we also evaluate the false-alarm probability for a fixed detection probability, which is based on the traditional Neyman-Pearson rule [11].

\section{B.Energy Ratio Algorithm}

Spectrum monitoring algorithm uses the secondary user physical layer model to investigate and verify the algorithm. The data coming from the source is firstly segmented into blocks where each block is randomized, channel encoded, and interleaved separately, at the transmitter side. The interleaving is followed by data modulation by a constellation mapper. The frequency domain OFDM frame is a combination of one or more training symbols or preambles that are used for both time and frequency synchronization at the receiver side, The modulated data and The BPSK modulated pilots which are used for data-aided synchronization algorithms employed by the receiver. If $N s$ denotes the number of sub-carriers per one OFDM symbol Each $N s$ encoded complex data symbols generated by the frame builder are used to construct one OFDM symbol by employing the IDFT block that is used to synthesize the OFDM symbol. The $\mathrm{m}^{\text {th }}$ symbols', $\mathrm{n}^{\text {th }}$ time-domain sample of can be expressed as given below, where $C(k, m)$ is the modulated data to be transmitted on the $\mathrm{m}^{\text {th }}$ OFDMsymbol with the $\mathrm{k}^{\text {th }}$ sub-carrier.

$$
\mathrm{s}(\mathrm{n}, \mathrm{m})=\frac{1}{\sqrt{\mathrm{N}_{\mathrm{s}}}} \sum_{\mathrm{k}=-\frac{\mathrm{N}_{\mathrm{S}}}{2}}^{\frac{\mathrm{N}_{\mathrm{S}}}{2}-1} \mathrm{C}(\mathrm{k}, \mathrm{m}) \mathrm{e}^{\mathrm{j} 2 \pi \mathrm{kn} / \mathrm{N}_{\mathrm{s}}}
$$

The last $\mathrm{Ng}$ samples of the time domain OFDM symbol are copied to the beginning of the symbol to form a guard time or cyclic prefix. This is to reduce the effect of Inter-Symbol Interference (ISI). The period of a OFDM block is $T s=(N s+N g) / F s$ where $F s$ is the sampling frequency. The inverse blocks are applied at the receiver side. The cyclic prefix is removed after timing synchronization and frequency synchronization. Timing synchronization includes frame detection, start of symbol timing and SFO estimation and compensation. Frequency synchronization includes CFO estimation and correction. Through an Ns point DFT the received OFDM symbol is transformed again into the frequency domain. This is followed by channel estimation and equalization of the received data. The demapper then maps the complex data output to bits. Now to recover the original source bits apply De-interleaving, decoding, and De-randomization to the received block. In this model the master node constructs OFDM frames in the downlink path. The frames are constructed such that the data sub-carriers are allocated in time and frequency for different users based on a predefined scheduling technique but the same pilots are transmitted to all slaves. Orthogonal Frequency Division Multiple Access (OFDMA) is assumed to divide the spectrum and the time into distinct and non-overlapping channels for different slaves, for the return path so that interferences between the slaves is avoided. The timing of each slave is completely controlled by the fusion node. This is done by allowing the slave know the required time advance or delay, so that the combined signal from all slaves seem to be synchronized at the fusion node receiver.

Here, the fusion node can convert the signal back to the frequency domain to extract the data and control information from different slaves. It is assumed that the slaves can send important information such as spectrum monitoring decisions and channel state information over a logical control channel in the return path. Based on the received monitoring decisions the master node can apply a majority rule and decide whether to stop transmission or not.

Before the IDFT, a number of tones are reserved for the spectrum monitoring purposes on the timefrequency grid of the OFDM frame. Such tones are reserved for the whole time except the time of the training symbols not to change the preamble waveform, which is used for synchronization at the receiver. The OFDM frame used is shown in Fig 3. 


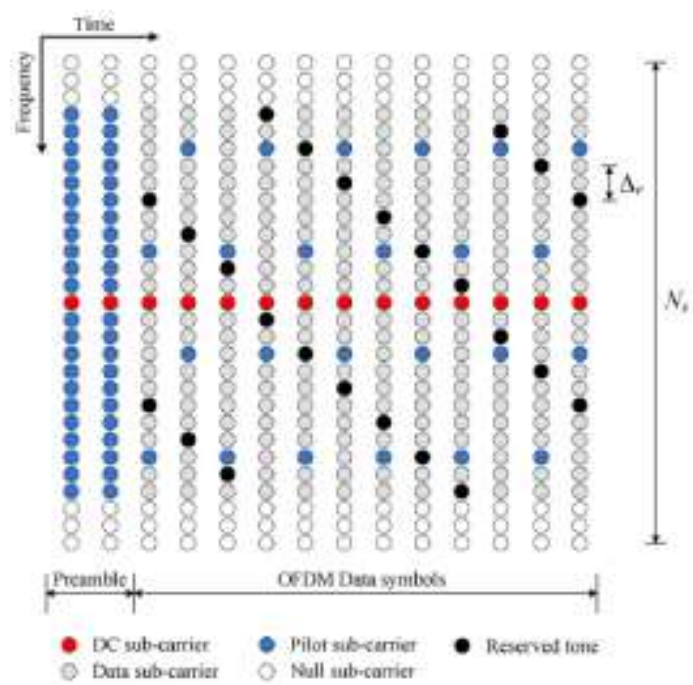

Fig. 3. Time-frequency allocation for one OFDM frame to explore different sub-carrier types [1]

The reserved tones are allocated dynamically for their indices to span the whole band when successive OFDM symbols are considered in time. Every OFDM symbol, the tones are advanced by $\Delta_{r}$ positions. The spanning starts again from the first subcarrier, as the last index of the available subcarriers is reached. The reserved tone sequence injected to the energy ratio spans the whole band for small values of $\Delta_{r}$. This scheduling is followed due to the following reasons.Firstly due to the use of OFDM the primary user may have some spectrum holes and the algorithm fails when the reserved tones from the SU are synchronized with those spectrum holes in the PU side. Whereas when the PU uses a traditional single carrier modulation technique like QAM, then PU signal has a flat spectrum over the entire band and therefore this issue does not have a harm effect on the algorithm. Secondly, the primary to secondary channel may introduce notch characteristics to the narrow band occupied by the reserved tones. This results in detecting lower primary power level, which is referred to the narrow band problem. Therefore to mitigate the channel effect and to protect the reserved tones from falling into primary holes reserved tones are rescheduled by changing the value of $\Delta_{r}$ over time. All SUs should know the code for this scheduling in prior.

The secondary user can monitor the band and test the primary user appearance, based on the signal on the reserved tones at the receiver. The traditional radiometer can be used to measure the primary signal power and the secondary noise power by accumulating the energy of those reserved tones. As a result, the primary signal power can be detected if this energy exceeds a predefined threshold. As the spectral leakage of the neighboring sub-carriers will affect the energy at the reserved tones even for no in-band primary signal this approach does not guarantee the primary user detection [10].Thus it leads to another decision making criterion that has a powerful immunity for this power leakage. This can also overcome the ICI resulted from the residual CFO and SFO errors, and even the effect of NBI. Fig 4. Illustrates the overall energy ratio algorithm.

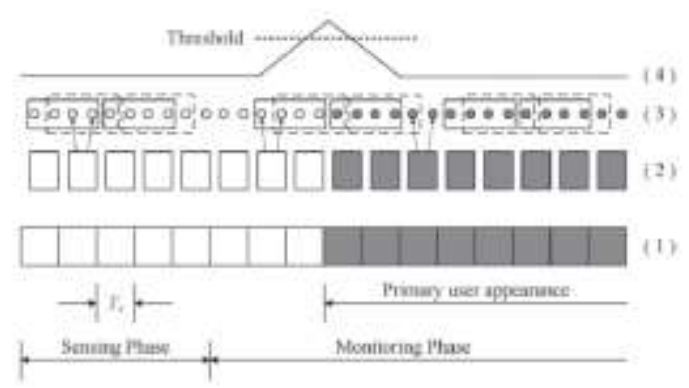

Fig. 4. Energy ratio processing details. (1) The time domain sequence for the OFDM blocks. (2) Frequency domain samples. (3) Reserved tones processing with two sliding windows for $N R T=2$ and $N=4$. (4) Decision making variable, $X k \cdot[1]$

Assume that the primary signal appears after some time during the monitoring phase. The reserved tones from different OFDM symbols are combined to form one sequence of complex samples, after CP removal and frequency domain processing on the received signal, at the secondary receiver. Over the reserved tone 
sequence in the time direction two consecutive equal-sized sliding windows are passed. The energy of the samples that fall in one window is calculated and the ratio of the two energies is taken as the decision making variable and therefore it's named as energy ratio algorithm.

The aim of the algorithm is to check the change in variance on the reserved tones over time. Mathematically let $Z i$ be the $i$ th sample of the reserved tone sequence. Now define the decision making variable, $X k$, as the ratio of the energy of the second window, $U k$, and the energy of the first window, $V k$ as given by (2).Where $N$ is the number of samples per window and $k$ is an integer such that $k=1,2,3, \ldots$

$$
X_{K}=\frac{U_{K}}{V_{K}}=\frac{\sum_{i N+k}^{2 N+k-1}\left|Z_{i}\right|^{2}}{\sum_{i=k}^{N+k-1}\left|Z_{i}\right|^{2}}
$$

The energy ratio algorithm starts the reserved tones processing from the beginning of the sensing phase. Therefore the decision making variable is calculated during both sensing and monitoring phases. But the decisions are provided only during the monitoring phase. When the decision from the spectrum sensing algorithm is that the PU is inactive during the sensing phase, the energy ratio algorithm has been properly calibrated to be able to detect the appearance of the PU during monitoring phase. That means both sliding windows are filled with pure unwanted signals. The receiver monitors the reserved tones by evaluating the parameter, $X k$, during the monitoring phase. When it exceeds a certain threshold, the secondary user assumes that there is a power change on the reserved tones which is perhaps due to the primary user appearance and it is time to vacate the band. When it is within a threshold value the secondary user can continue transmission. The energy of each window involves only the strength of the unwanted signals including the noise, the leakage from the neighboring sub-carriers, and the effects of ICI produced by the residual synchronization errors when there is no primary user in band. The ratio will be very close to unity since the strength of the unwanted signals does not offer significant changes over time, when $N$ is large enough.

When the primary user appears the second window will have two types of signaling which are the primary user interference and the unwanted signals. Whereas the first window will only maintain the unwanted signals without the primary user interference. Hence the ratio of the two energies will result in much higher values when compared to one. Of course, the ratio value will depend on the primary user power. As the two windows slide again, the ratio is close to unity since the primary signal plus the unwanted signals will be observed by the two windows and the decision making variable returns to the initial state. Thus the decision variable produces a spike when the primary user is detected. Else it changes very slowly maintaining the energy ratio close to one. This approach can resist the different impairments involved in the received signal but it leads to reduction in the throughput of the secondary user by the ratio of the number of reserved tones to the number of useful tones. But, this reduction can be overcome since OFDM systems allow adaptive modulation where good conditioned sub-carriers are loaded with higher modulation order.

Assume the primary user should appear at the boundaries of the OFDM blocks. So, when the primary user is active the reserved tones should have the full power, which is supposed to be for those sub-carrier indices, of the primary user. But practically, the primary user may appear any time within any OFDM block in the monitoring phase. Two effects have to be considered in this case. (1) The FFT window applied by the SU receiver will have a time-shifted version of the PU signal and that involves a phase rotation to the PU subcarriers. The phase shift is acceptable to happen with no effect on the algorithm since the energy is the useful parameter for this algorithm. (2) Since part of the signal is truncated, the power on the reserved tones will not have the full power transmitted by the primary user on those sub-carriers. But the next OFDM symbol will have that full power. If the PU power is large enough, then the reserved tones form the first OFDM symbol, in which PU signal appears, are considered to be full, similar to near far problem. Else, the reserved tones from this OFDM symbol are considered as noise if $N \gg N R T$.

\section{Summary And Conclusion}

In this survey we have seen different spectrum monitoring techniques for OFDM based Cognitive radio networks. In the receiver statistics method the cognitive radios of the secondary network to perform spectrum monitoring during the reception of packets. These protocols reduce the frequency with which traditional spectrum sensing must be performed, therefore greater spectral efficiency is obtained by the secondary network. When the receiver statistics suggest that a transmission from a primary user may have emerged, then the secondary user's session is suspended temporarily while more. This also decreases the time required to detect the emergence of the primary signal accurate traditional spectrum sensing is employed, compared to traditional spectrum monitoring techniques. REC-based spectrum monitoring by a single secondary receiver will not be enough if the primary signal is very weak (i.e., if SPR is very large). Instead of that, either cooperative monitoring among multiple geographically distributed secondary receivers or a combination of spectrum monitoring and traditional spectrum sensing must be employed. These protocols have very low complexity and can be employed even in systems that rely primarily on traditional spectrum sensing. Like all protocols, these 
protocols for spectrum monitoring have backup modes for unanticipated circumstances. For example, when poor or highly variable channel conditions cause spectrum monitoring to produce several consecutive false alarms, then the protocol resorts to traditional spectrum sensing temporarily until the channel improves. Large variations in the REC, IC, or other receiver statistics from packet to packet provide an indication of time-varying disturbances on the channel, and they suggest that perhaps the backup mode should be employed temporarily. Whereas a return to smaller variations in the receiver statistics is an indication that spectrum monitoring during packet reception can be resumed in the secondary network.

Another spectrum monitoring algorithm that can sense the reappearance of the primary user during the secondary user transmission is the energy ratio algorithm. This is designed for OFDM systems such as Ecma392 and IEEE802.11af systems. The detection performance is superior than the receiver statistics method. For computational complexity, the energy ratio architecture is investigated where it was shown that it requires only about double the complexity of the conventional energy detector. This spectrum monitoring algorithm can greatly improve the performance of OFDM-based cognitive networks by improving the detection performance with a very limited reduction in the secondary network throughput.

\section{References}

[1]. Abdelmohsen Ali and Walaa Hamouda, "Spectrum Monitoring Using Energy Ratio Algorithm for OFDM-Based Cognitive Radio Networks," IEEE Trans. wireless communications vol. 14, no. 4,April 2015.

[2]. S. W. Boyd, J. M. Frye, M. B. Pursley, and T. C. Royster, "Spectrum monitoring during reception in dynamic spectrum access cognitive radio networks," IEEE Trans. Commun., vol. 60, no. 2, pp. 547-558, Feb. 2012.

[3]. H. Mahmoud, T. Yucek, and H. Arslan, "OFDM for cognitive radio: Merits and challenges," IEEE Wireless Commun., vol. 16, no.2, pp. 6-15, Apr. 2009.

[4]. W. S. Jeon, D. G. Jeong, J. A. Han, G. Ko, and M. S. Song, "An efficient quiet period management scheme for cognitive radio systems," IEEE Trans. Wireless Commun., vol. 7, no. 2, pp. 505-509, Feb. 2008.

[5]. S. H. Hwang and M. J. Rim, “Adaptive operation scheme for quiet period in IEEE 802.22 system," in Proc. ICTC, Sep. 2011, pp. $482-484$.

[6]. D. Cabric, S. M. Mishra, and R. W. Brodersen, "Implementation issues inspectrum sensing for cognitive radios," in Proc. Conf. Rec. 38th Asilomar Conf. Signals, Syst. Comput., Nov. 2004, vol. 1, pp. 772-776.

[7]. D. Chen, J. Li, and J. Ma, "In-band sensing without quiet period in cognitive radio," in Proc. IEEE WCNC, 2008, pp. 723-728.

[8]. W. Hu et al., "Cognitive radios for dynamic spectrum access-Dynamic frequency hopping communities for efficient ieee 802.22 operation," IEEE Commun. Mag., vol. 45, no. 5, pp. 80-87, May 2007.

[9]. R. Saifan, A. Kamal, and Y. Guan, "Efficient spectrum searching and monitoring in cognitive radio network," in Proc. IEEE 8th Int. Conf. MASS, 2011, pp. 520-529.

[10]. T. Ihalainen, A. Viholainen, T. Stitz, and M. Renfors, "Spectrum monitoring scheme for filter bank based cognitive radios," in Proc. Future Netw. Mobile Summit, Jun. 2010, pp. 1-9.

[11]. S. M. Kay, Fundamentals of Statistical Signal Processing: Detection Theory. Prentice Hall, 1998.

[12]. S. Haykin, "Cognitive radio: Brain-empowered wireless communications," IEEE J. Sel. Areas Commun., vol. 23, no. 2, pp. 201220, Feb. 2005.

[13]. A. Ghosh and W. Hamouda, "Cross-layer antenna selection and channel allocation for MIMO cognitive radios," IEEE Trans. Wireless Commun., vol. 10, no. 11, pp. 3666-3674, Nov. 2011.

[14]. X. Gong, S. Vorobyov, and C. Tellambura, "Optimal bandwidth and power allocation for sum ergodic capacity under fading channels in cognitive radio networks," IEEE Trans. Signal Process., vol. 59, no. 4, pp. 1814-1826, 2011.

[15]. J.-S. Urn, S.-H. Hwang, and B.-J. Jeong, "A comparison of PHY layer on the Ecma-392 and IEEE 802.11af standards," in Proc. Int. Conf. Cognitive Radio Oriented Wireless Netw. Commun., Jun. 2012, pp. 313-319.

[16]. T. Ihalainen, A. Viholainen, T. Stitz, and M. Renfors, "Spectrum monitoring scheme for filter bank based cognitive radios," in Proc. Future Netw. Mobile Summit, Jun. 2010, pp. 1-9.

[17]. F. Digham, M.-S. Alouini, and M. K. Simon, "On the energy detection of unknown signals over fading channels," in Proc. IEEE Int. Conf. Commun., 2003, vol. 5, pp. 3575-3579.

[18]. A. Ghosh and W. Hamouda, "On the performance of interference aware cognitive ad-hoc networks," IEEE Commun. Lett., vol. 17, no. 10 , pp. $1952-1955$, Oct. 2013.

[19]. D. Galda and H. Rohling, "Narrow band interference reduction in ofdm based power line communication systems," in Proc. IEEE ISPLC, Apr. 2001, pp. 345-351

[20]. S. Brandes, I. Cosovic, and M. Schnell, "Reduction of out-of-band radiation in ofdm systems by insertion of cancellation carriers," IEEE Commun. Lett., vol. 10, no. 6, pp. 420-422, Jun. 2006.

[21]. M. Ma, X. Huang, B. Jiao, and Y. Guo, "Optimal orthogonal precoding for power leakage suppression in DFT-based systems," EEE Trans. Commun., vol. 59, no. 3, pp. 844-853, 2011. 